\title{
A TURBULENCE MODEL TAKING INTO ACCOUNT THE LONGITUDINAL FLOW INHOMOGENEITY IN MIXING LAYERS AND JETS
}

\author{
A. I. Troshin \\ Central Aerohydrodynamic Institute (TsAGI) \\ 1 Zhukovsky Str., Zhukovsky, Moscow Region 140180, Russia
}

\begin{abstract}
The problem of potential core length overestimation of subsonic free jets by Reynolds-averaged Navier-Stokes (RANS) based turbulence models is addressed. It is shown that the issue is due to the incorrect velocity profile modeling of the jet mixing layers. An additional source term in $\omega$ equation is proposed which takes into account the effect of longitudinal flow inhomogeneity on turbulence in mixing layers. Computations confirm that the modified Speziale-Sarkar-Gatski/LaunderReece-Rodi-omega (SSG/LRR- $\omega$ ) turbulence model correctly predicts the mean velocity profiles in both initial and far-field regions of subsonic free plane jet as well as the centerline velocity decay rate.
\end{abstract}

\section{INTRODUCTION}

Many RANS-based turbulence models overestimate the potential core length of subsonic free jets [1]. Analysis shows that this issue is due to incorrect velocity profile modeling of the jet mixing layers $[2,3]$. To solve this problem, a correction which increases the turbulence diffusion intensity near the jet axis is proposed in [1]. Unfortunately, this correction is not Galilean invariant. In [3], an invariant correction is suggested which enhances the turbulence diffusion at the edges of turbulent regions and recovers the velocity profile of a single stream mixing layer.

In this paper, a further investigation is conducted. Another way to reduce the jet potential core length in computations is proposed which is not connected to the modification of turbulence diffusion coefficients. Instead, an additional source term in characteristic turbulence frequency equation is proposed. The differential Reynolds stress model (DRSM) SSG/LRR- $\omega$ [4] is taken as the basis for improvement. This is a modern DRSM with a great potential in modeling complex problems with nonequilibrium turbulence, separation, secondary flows, and streamline curvature. The SSG/LRR- $\omega$ model uses a blending function to

(C) The authors, published by EDP Sciences. This is an Open Access article distributed under the terms of the Creative Commons Attribution License 4.0 (http://creativecommons.org/licenses/by/4.0/). 
switch between the near-wall and free turbulent coefficient values. In the present study, only "free turbulent part" of the model is modified.

The structure of the paper is as follows. In section 2, the equations of the original SSG/LRR- $\omega$ model are quoted as well as the results of self-similar computations of mixing layers and a plane jet. In section 3, a calibration of model coefficients is conducted using the temporal mixing layer data. In section 4 , an additional source term in $\omega$ equation is developed which improves the spatial mixing layer velocity profile. Finally, in section 5, the computations using the complete Reynolds equation system closed by the modified turbulence model are presented and the improvements are shown which result from the changes made.

\section{SSG/LRR- $\omega$ MODEL PERFORMANCE IN FREE TURBULENT FLOWS}

The original SSG/LRR- $\omega$ model equations are as follows [4]:

$$
\begin{aligned}
& \frac{\partial \bar{\rho} R_{i j}}{\partial t}+\frac{\partial}{\partial x_{k}}\left(\bar{\rho} R_{i j} \bar{u}_{k}-\mu \frac{\partial R_{i j}}{\partial x_{k}}-C_{R} \bar{\rho} \frac{k}{\varepsilon} R_{k l} \frac{\partial R_{i j}}{\partial x_{l}}\right)=\bar{\rho}\left(P_{i j}+\Pi_{i j}-\frac{2}{3} \varepsilon \delta_{i j}\right) \\
& \frac{\partial \bar{\rho} \omega}{\partial t}+\frac{\partial}{\partial x_{k}}\left(\bar{\rho} \omega \bar{u}_{k}-\mu \frac{\partial \omega}{\partial x_{k}}-C_{\omega} \bar{\rho} \frac{k^{2}}{\varepsilon} \frac{\partial \omega}{\partial x_{k}}\right) \\
&=\bar{\rho} \frac{\omega}{k}\left(C_{\omega 1} P-C_{\omega 2} \varepsilon\right)+\frac{\alpha_{d} C_{\omega}}{C_{\mu}} \frac{\bar{\rho}}{\omega} \max \left(\frac{\partial k}{\partial x_{k}} \frac{\partial \omega}{\partial x_{k}}, 0\right)
\end{aligned}
$$

Hereinafter, summation over repeated indices is adopted; $R_{i j}=\overline{u_{i}^{\prime} u_{j}^{\prime}}$ is the Reynolds stress tensor; $\omega$ is the characteristic turbulence frequency; $k=R_{i i} / 2$ is the turbulence kinetic energy; and $\varepsilon=C_{\mu} k \omega$ is its dissipation rate $\left(C_{\mu}=0.09\right)$. Reynolds stress production is computed according to the exact formula:

$$
P_{i j}=-R_{i k} \frac{\partial \bar{u}_{j}}{\partial x_{k}}-R_{j k} \frac{\partial \bar{u}_{i}}{\partial x_{k}} .
$$

Turbulence kinetic energy production is $P=P_{i i} / 2$. Redistribution term $\Pi_{i j}$ is computed using SSG model [5] in free turbulent regions and LRR model with coefficients proposed by Wilcox [6] in near-wall regions. Introducing the Reynolds stress anisotropy tensor $a_{i j}=\left(R_{i j}-2 k \delta_{i j} / 3\right) / k$, strain rate tensor $\bar{S}_{i j}=\left(\partial \bar{u}_{i} / \partial x_{j}+\partial \bar{u}_{j} / \partial x_{i}\right) / 2$, and vorticity tensor $\bar{\Omega}_{i j}=\left(\partial \bar{u}_{i} / \partial x_{j}-\partial \bar{u}_{j} / \partial x_{i}\right) / 2$, it can be written as follows: 
Table 1 The original SSG/LRR- $\omega$ model coefficients

\begin{tabular}{lllllllllllll}
\hline Turbulence & $C_{\omega 1}$ & $C_{\omega 2}$ & $C_{R}$ & $C_{\omega}$ & $\alpha_{d}$ & $C_{1}$ & $C_{1}^{*}$ & $C_{2}$ & $C_{3}$ & $C_{3}^{*}$ & $C_{4}$ & $C_{5}$ \\
\hline Near-wall & 0.5556 & 0.8333 & 0.0675 & 0.045 & 0 & 1.8 & 0 & 0 & 0.8 & 0 & $\frac{267}{275}$ & $\frac{159}{275}$ \\
Free & 0.44 & 0.92 & 0.22 & 0.077 & 2.0 & 1.7 & 0.9 & 1.05 & 0.8 & 0.65 & 0.625 & 0.2 \\
\hline
\end{tabular}

$$
\begin{aligned}
\Pi_{i j}= & -\left(C_{1} \varepsilon+C_{1}^{*} P\right) a_{i j}+C_{2} \varepsilon\left(a_{i k} a_{k j}-\frac{1}{3} a_{k l} a_{k l} \delta_{i j}\right)+\left(C_{3}-C_{3}^{*} \sqrt{a_{k l} a_{k l}}\right) k \bar{S}_{i j} \\
& +C_{4} k\left(\bar{S}_{i k} a_{k j}+a_{i k} \bar{S}_{k j}-\frac{2}{3} a_{k l} \bar{S}_{k l} \delta_{i j}\right)+C_{5} k\left(\bar{\Omega}_{i k} a_{k j}-a_{i k} \bar{\Omega}_{k j}\right) .
\end{aligned}
$$

The coefficients of the model $V$ are determined as $V=V_{\text {wall }} F_{1}+V_{\text {free }}\left(1-F_{1}\right)$ where the blending function $F_{1}$ has the form $F_{1}=\tanh \zeta^{4}$,

$$
\zeta=\min \left[\max \left(\frac{\sqrt{k}}{C_{\mu} \omega d_{w}}, \frac{500 \mu}{\bar{\rho} \omega d_{w}^{2}}\right), \frac{4 k \omega}{\alpha_{d, \text { free } \max \left(\left(\partial k / \partial x_{k}\right)\left(\partial \omega / \partial x_{k}\right), 0\right) d_{w}^{2}}}\right]
$$

with $d_{w}$ being the distance to the nearest wall.

Coefficient values for near-wall and free turbulent regions are presented in Table 1.

This turbulence model has been used to compute three free turbulent incompressible self-similar flows. The first is temporal mixing layer which forms between two parallel streams with almost equal velocities $\left|u_{2}-u_{1}\right| \ll u_{1}+u_{2}$ [7]. In the coordinate frame moving with velocity $\left(u_{1}+u_{2}\right) / 2$ and $y$ axis normal to the flows, the mixing layer is symmetrical relative to its center, longitudinal gradients of every flow variable, and transverse velocity component are negligible and mixing zone thickness linearly grows with time. To compute the self-similar temporal mixing layer velocity profile, it is sufficient to solve only the longitudinal momentum equation supplied by the shear stress profile:

$$
\frac{\partial \bar{u}}{\partial t}+\frac{\partial R_{x y}}{\partial y}=0
$$

Let us introduce the self-similar variables $\tau=t$ and $\eta=y /\left(u_{0} t\right)$, where $u_{0}>0$ is the velocity of either stream in the chosen coordinate frame, and denote $f(\eta)=\bar{u}(t, y) / u_{0}$ and $r_{i j}(\eta)=R_{i j}(t, y) / u_{0}^{2}$. In these variables, Eq. (6) takes the form:

$$
-\eta f^{\prime}+r_{x y}^{\prime}=0 \text {. }
$$

Equations (1) and (2) used to close this equation are converted similarly.

Two other flows are the spatial single-stream mixing layer (Fig. 1a) and the far-field region of a free plane jet (Fig. 1b). Both of them are inhomogeneous in 


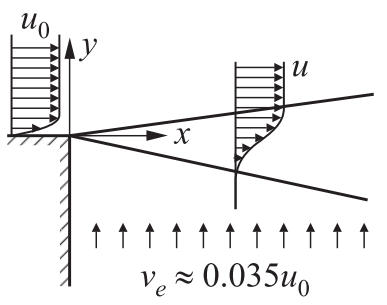

(a)

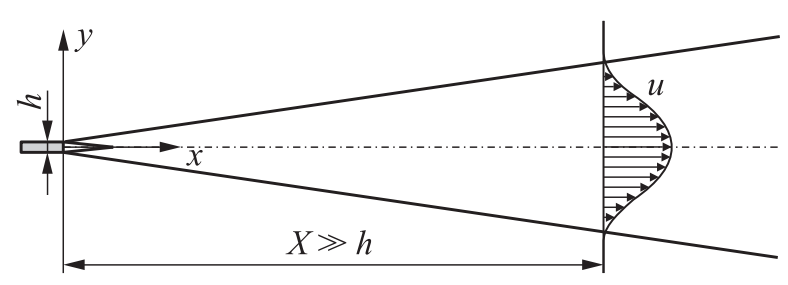

(b)

Figure 1 Flow field scheme of single-stream mixing layer $(a)$ and free plane jet far field $(b)$

longitudinal direction and contain nonzero transverse (ejection) velocity component $v_{e}$.

The equation system describing these flows is the following:

$$
\begin{array}{r}
\frac{\partial \bar{u}}{\partial x}+\frac{\partial \bar{v}}{\partial y}=0 ; \\
\bar{u} \frac{\partial \bar{u}}{\partial x}+\bar{v} \frac{\partial \bar{u}}{\partial y}+\frac{1}{\bar{\rho}} \frac{\partial \bar{p}}{\partial x}+\frac{\partial R_{x x}}{\partial x}+\frac{\partial R_{x y}}{\partial y}=0 ; \\
\bar{u} \frac{\partial \bar{v}}{\partial x}+\bar{v} \frac{\partial \bar{v}}{\partial y}+\frac{1}{\bar{\rho}} \frac{\partial \bar{p}}{\partial y}+\frac{\partial R_{x y}}{\partial x}+\frac{\partial R_{y y}}{\partial y}=0 .
\end{array}
$$

Again, let us use the self-similar variables $\xi=x$ and $\eta=y / x$ and denote $\bar{u}(x, y)=A x^{b} f(\eta), \bar{v}(x, y)=A x^{b} g(\eta), R_{i j}(x, y)=B x^{2 b} r_{i j}(\eta), \omega(x, y)$ $=C x^{3 b-1} w(\eta)$ and $F=\left(f^{2}+g^{2}\right)^{1 / 2}$, and $\kappa=r_{i i} / 2$. For the mixing layer, $b=0$. For the plane jet, $b=-1 / 2$. Let us use the only simplification of (8)-(10):

$$
\frac{1}{\bar{\rho}} \frac{\partial \bar{p}}{\partial x}=-\frac{\partial R_{y y}}{\partial x} .
$$

No other assumptions leading to "thin-shear-flow" approximation are used because they can distort the solution by as much as $12 \%$ [8].

In $(\xi, \eta)$-variables, Eqs. (8)-(10) read:

$$
\begin{array}{r}
b f-\eta f^{\prime}+g^{\prime}=0 \\
b f^{2}+(g-\eta f) f^{\prime}+2 b\left(r_{x x}-r_{y y}\right)-\eta\left(r_{x x}^{\prime}-r_{y y}^{\prime}\right)+r_{x y}^{\prime}=0 .
\end{array}
$$

Equations (1) and (2) are converted similarly.

A program has been implemented which solves Eqs. (7), (11), and (12) using time marching. An explicit second-order spatial scheme is adopted. At the external boundaries, very weak isotropic turbulence is specified: $r_{i j \infty}=2 \kappa_{\infty} \delta_{i j} / 3$, 
$\kappa_{\infty} \ll \kappa_{\max }$, and $w_{\infty} \ll w_{\max }$ where $\kappa_{\max }$ and $w_{\max }$ are the maximal values of $\kappa$ and $w$ in the whole computational domain. It is checked that the solutions obtained within turbulent zones are insensitive to the $\kappa_{\infty}$ and $w_{\infty}$ chosen as described.

A mesh convergence study has been performed, resulting in the decision to use the meshes containing 150 nodes within the turbulent zone.

In Fig. 2, velocity profiles obtained with SSG/LRR- $\omega$ model are compared with the experimental data (see [9] for temporal mixing layer, the same as in [3] for the other flows). The temporal mixing layer is plotted using the scaled coordinate $\eta^{*}=f^{\prime}(0) \eta$ which makes the velocity slope in the center equal 1 . From the data presented, the following conclusions can be made:

(1) SSG/LRR- $\omega$ model predicts too sharp turbulent zone boundaries. This problem is evident in all the flows considered;

(2) the low-velocity part of the spatial mixing layer is wider than the highvelocity part. Note that this shortcoming is inherent to virtually all differential turbulence models [3,6]; and

(3) spatial mixing layer width is underestimated.

Sharp turbulent zone boundaries is a feature of the solution structure at the interface between turbulent and nonturbulent flow regions (TNT interface) [10]. Depending on turbulent diffusion coefficients $C_{R}, C_{\omega}$, and $\alpha_{d}$, different power-law profiles of velocity and turbulence variables can be obtained near a TNT interface. The original SSG/LRR- $\omega$ model produces approximately linear velocity profiles. They can be smoothed by calibrating the turbulent diffusion coefficients. In the following section, this is done using the simplest flow considered, namely, temporal mixing layer.

\section{COEFFICIENTS CALIBRATION USING TEMPORAL MIXING LAYER DATA}

Three series of temporal mixing layer computations have been conducted, in which coefficients $C_{R}, C_{\omega}$, and $\alpha_{d}$ have been successively varied. The influence of these coefficients is shown in Fig. 3.

Coefficient $C_{R}$ changes the velocity profile very strongly. Its influence appears not only near the turbulent zone boundaries, but spreads deeply inside it. As a result, it is impossible to obtain an accurate velocity profile which would fully conform to the experimental data by varying $C_{R}$ only.

Coefficient $C_{\omega}$ determines the velocity profile behavior only near the turbulent zone boundaries and there is "saturation" at high $C_{\omega}$ values, when velocity profile becomes practically straight and does not change any further. 


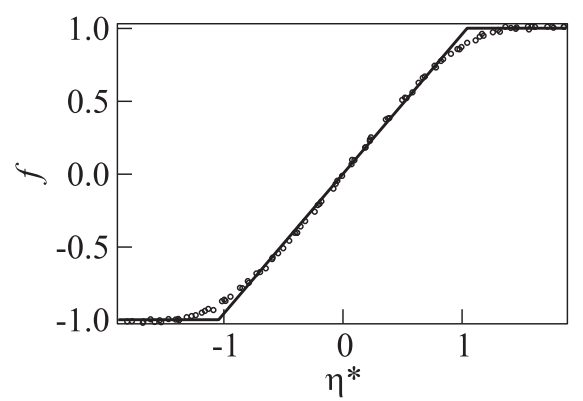

(a)

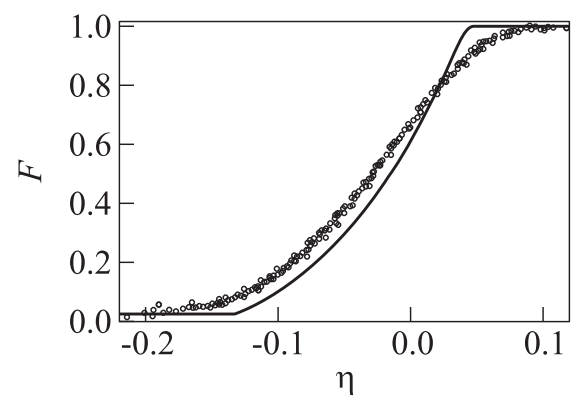

(b)

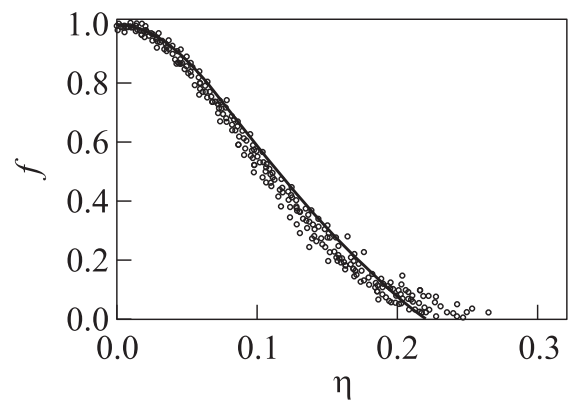

(c)

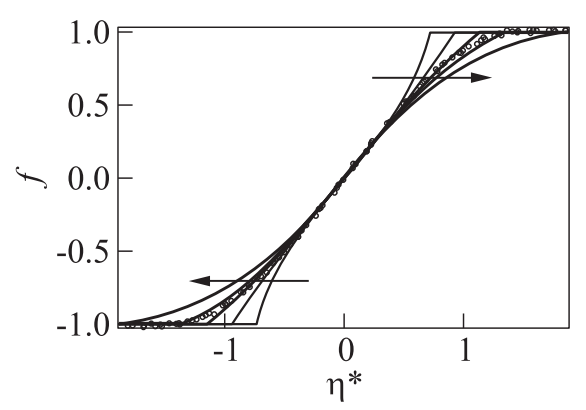

(a)

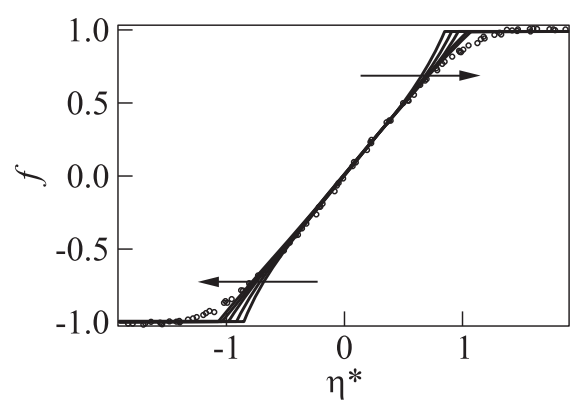

(b)

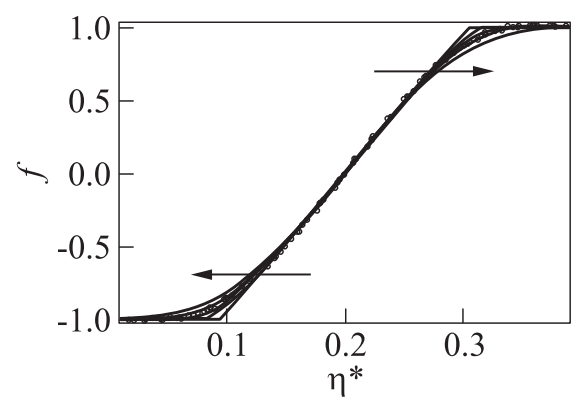

(c)

Figure 2 Self-similar solutions obtained with the original SSG/LRR- $\omega$ model: (a) temporal mixing layer; (b) single-stream mixing layer; and (c) free plane jet far field

Figure 3 Influence of the coefficients $C_{R}(a), C_{\omega}(b)$, and $\alpha_{d}(c)$. Arrows indicate the direction of coefficient increase 
Coefficient $\alpha_{d}$ plays crucial role in velocity profile shaping. It influences its boundaries allowing to make it either sharp or very smooth.

To find the family of coefficient sets giving the temporal mixing layer velocity profile lying in the experimental data range, a series of parametric computations has been conducted.

In each computation, fixed values of $C_{R}$ and $C_{\omega}$ have been set, and $\alpha_{d}$ adjusted so that $\eta_{0.99}^{*}$ coordinate (a boundary point where $f=0.99$ ) would be equal, according to experiments, 1.36. As a result, for the val-

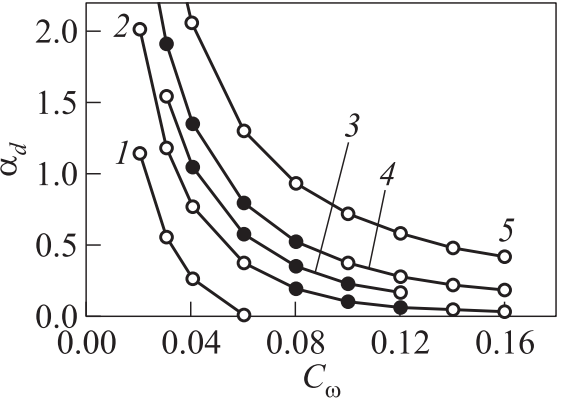

Figure 4 A diagram of SSG/LRR- $\omega$ model calibration using the temporal mixing layer data: $1-C_{R}=0.14 ; 2-0.18 ; 3-0.20$; $4-0.22 ;$ and $5-C_{R}=0.26$ ues $C_{R}=0.14,0.18,0.20,0.22$, and 0.26 , the curves $\alpha_{d}\left(C_{\omega}\right)$ have been obtained, points on which correspond to temporal mixing layer velocity profiles with correct boundary positions. The diagram consisting of these curves is shown in Fig. 4.

Since the condition $\eta_{0.99}^{*}=1.36$ does not fully determine the velocity profile, among the coefficient sets found, there are ones which give distorted velocity profiles in the regions between the center of the turbulent zone and its boundaries. The region of coefficient sets giving velocity profiles entirely matching the experimental data is marked on the diagram with black circles. The distribution of the black circles on the diagram indicates that in the $0.18 \leq C_{R} \leq 0.22$ range, accurate temporal mixing layer modeling is possible.

\section{TAKING INTO ACCOUNT THE LONGITUDINAL FLOW INHOMOGENEITY}

The first step to calibrate the coefficients of the SSG/LRR- $\omega$ model using the spatial single-stream mixing layer data is to determine the $\alpha_{d}$ value provided $C_{\omega 1}=0.48$ and $C_{\omega 2}=0.86$ so that the mixing layer thickness $D_{0.1}$ computed by the points where $F=0.1$ and 0.9 would be equal to the experimental value 0.165 . The $C_{\omega 1}$ and $C_{\omega 2}$ values are taken from the middle of the "generally accepted" ranges $0.44 \leq C_{\omega 1} \leq 0.52$ and $0.80 \leq C_{\omega 2} \leq 0.92$ [6]. Coefficient $\alpha_{d}$ appeared to be equal to 0.566 . From the diagram (see Fig. 4), it follows that $C_{R}=0.20$ and $C_{\omega}=0.0593$. The velocity profile of single-stream mixing layer computed with these coefficient values is presented in Fig. $5 a$ (curve 1). It has the drawback which has already been noticed: the turbulent zone spreads too strongly towards 


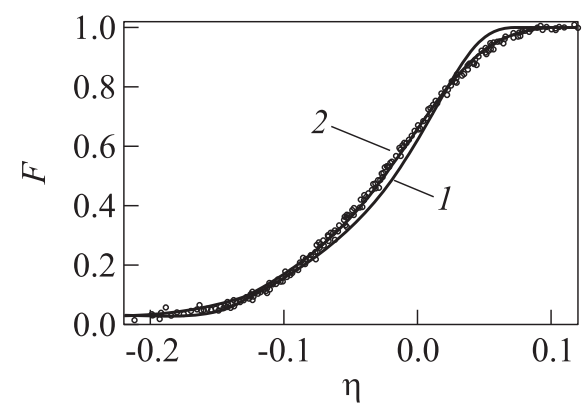

(a)

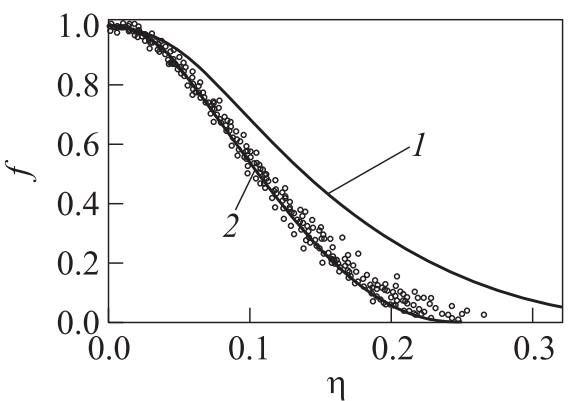

(b)

Figure 5 Velocity profile of single-stream mixing layer $(a)$ and free plane jet far field (b) before (1) and after (2) the introduction of the extra source term $I_{\omega}^{(0)}(a)$ or $I_{\omega}(b)$ in the SSG/LRR- $\omega$ turbulence model with the modified coefficient values

the low-velocity region of the mixing layer and too weakly towards the highvelocity region. The potential core length estimation using this velocity profile is $L_{\text {ini }}=8.46$ which is about 1.5 times higher than the experimental values $5 \leq L_{\text {ini }} \leq 6$.

It turns out that a turbulence model, which correctly describes the temporal mixing layer, at the same time strongly distorts the single-stream mixing layer. Hence, this distortion is due to the effects absent in the temporal mixing layer: longitudinal gradients of velocity and turbulence variables and transverse velocity component connected to the gas ejection into the turbulent zone. To author's knowledge, the only attempt to explicitly take into account these effects in a turbulence model is paper [11] in which it is suggested to include an extra source term in the $\varepsilon$ equation:

$$
\frac{\partial \varepsilon}{\partial t}+\frac{\partial}{\partial x_{k}}(\cdots)=\frac{\varepsilon}{k}\left(\cdots+C_{\varepsilon 4} \frac{k^{5 / 2}}{\varepsilon} \nabla|\vec{V}| \cdot \nabla \gamma\right)
$$

where $\gamma$ is the intermittency factor defined as the probability of the point in space to be in the turbulent region at a given time [12].

It increases the production of $\varepsilon$ at the low-velocity boundary of a mixing layer and decreases it at the high-velocity boundary which reduces the turbulent zone spread into $\eta<0$ region and enhances - into $\eta>0$ region.

In this paper, another source term is proposed which does not require the intermittency factor and is strictly Galilean invariant. In accordance with the observations made above, it depends on the longitudinal gradients of mean velocity and turbulence variables and on ejection velocity. Like the extra source term in Eq. (13), it should behave as an "odd function" (i. e., to be of different signs in the regions $\eta<0$ and $\eta>0$ ). This source term is included in the $\omega$ equa- 
tion which is empirical in nature and allows modifications. On the other hand, the $R_{i j}$ equation (1) is left unchanged because it has an exact form derived from the Navier-Stokes eqation system.

To obtain the most general form of this term, let us restrict ourselves by using only mean velocity, $k$ and $\omega$ gradients, but not individual $R_{i j}$ components. If necessary, these restrictions would permit us to adapt the source term to the eddy viscosity models in the future. The second derivatives are not involved for simplicity. Velocity vector itself is not used as it is not the Galilean invariant.

Using the above mentioned parameters, it is possible to form the following dimensionless aggregates:

$$
\begin{array}{ll}
N_{k k}=\frac{1}{k \omega^{3}} \frac{\partial \bar{u}_{i}}{\partial x_{j}} \frac{\partial k}{\partial x_{i}} \frac{\partial k}{\partial x_{j}} ; & N_{k \omega}=\frac{1}{\omega^{4}} \frac{\partial \bar{u}_{i}}{\partial x_{j}} \frac{\partial k}{\partial x_{i}} \frac{\partial \omega}{\partial x_{j}} \\
N_{\omega k}=\frac{1}{\omega^{4}} \frac{\partial \bar{u}_{i}}{\partial x_{j}} \frac{\partial \omega}{\partial x_{i}} \frac{\partial k}{\partial x_{j}} ; & N_{\omega \omega}=\frac{k}{\omega^{5}} \frac{\partial \bar{u}_{i}}{\partial x_{j}} \frac{\partial \omega}{\partial x_{i}} \frac{\partial \omega}{\partial x_{j}} .
\end{array}
$$

Velocity gradient norms are not used in the denominators of these formulas since they can vanish in the uniform flow outside the turbulent zones.

In homogeneous turbulence and in the temporal mixing layer, all the expressions $N_{k k}, N_{k \omega}, N_{\omega k}$, and $N_{\omega \omega}$ are identically zero. It can be shown that in self-similar spatial mixing layer, $N_{k k} \equiv 0$ and $N_{k \omega} \equiv 0$, but $N_{\omega k} \neq 0$ and $N_{\omega \omega} \neq 0$. Moreover, both $N_{\omega k}$ and $N_{\omega \omega}$ behave as an "odd function" relative to the mixing layer center.

Two extra source terms have been successively added to the $\omega$ equation:

$$
I_{\omega}^{(0)}=-C_{\omega 3} N_{\omega k} \omega^{2} ; \quad J_{\omega}^{(0)}=-C_{\omega 3}^{\prime} N_{\omega \omega} \omega^{2} .
$$

Negative signs in these formulas allow to use positive $C_{\omega 3}$ and $C_{\omega 3}^{\prime}$ values to get additional $\omega$ production at the low-velocity boundary of the mixing layer and $\omega$ dissipation at the high-velocity boundary. It is found that $I_{\omega}^{(0)}$ is much more effective than $J_{\omega}^{(0)}$ and with $C_{\omega 3} \geq 20$, it produces the mixing layer velocity profile entirely matching the experimental data. The $C_{\omega 3}=21$ value is chosen which gives the potential core length estimation $L_{\text {ini }}=5.80$ and mixing layer width $D_{0.1}=0.165$ without any extra turbulence model tuning. In Fig. $5 a$, velocity profiles are depicted without $I_{\omega}^{(0)}(1)$ and with it (2).

A shortcoming of the $I_{\omega}^{(0)}$ source term is that with $C_{\omega 3} \geq 22$, the solution becomes unphysical: near the high-velocity mixing layer boundary, a running turbulent front appears on which instability develops. Moreover, even with $C_{\omega 3}=21$ obtaining the stationary solution for the plane jet, far field becomes a challenge.

Analysis has shown that the dimensionless aggregate $N_{\omega k}$ which enters the $I_{\omega}^{(0)}$ does not exceed 0.01 in the core of the mixing layer, but near its boundary, $N_{\omega k}$ increases by several orders of magnitude. It is this uncontrollable 
growth of $N_{\omega k}$ which leads to the instability; consequently, $N_{\omega k}$ needs to be limited. The second reason for limiting is reduction of $I_{\omega}^{(0)}$ impact on the boundaries of turbulent zones, accurate description of which has been obtained earlier using the temporal mixing layer data.

Several modifications of $N_{\omega k}$ have been examined, which resulted in the following form of limiting:

$$
I_{\omega}^{(1)}=-C_{\omega 3} 0.03 \tanh \frac{N_{\omega k}}{0.03} \omega^{2} .
$$

With this limiting, the restriction $C_{\omega 3} \lesssim 21$ vanishes. To compensate for some loss of efficiency due to the limiting, $C_{\omega 3}$ has been increased to 22 . With the presented modification, the width of the mixing layer practically leaves unchanged $\left(D_{0.1}=0.164\right)$ and $L_{\mathrm{ini}}=5.88$. Velocity profile in the scale of Fig. $5 a$ is undistinguishable from the earlier obtained profile with $I_{\omega}^{(0)}$. Now, one can say that the goal of correct modeling of the spatial single stream mixing layer is reached.

The computation of the plane jet has showed that without $I_{\omega}^{(1)}$, jet halfwidth $\eta_{0.5}$ computed by the point where $f=0.5$ equals to 0.139 whereas mean experimental value is 0.105 . With $I_{\omega}^{(1)}$, it reduces to 0.098 , i. e., $I_{\omega}^{(1)}$ increases the dissipation in the plane jet too much.

The following solution of this issue is proposed. It has been noticed that in self-similar mixing layer, $N_{k \omega} \equiv 0$ but it is not zero in the plane jet. Let us use this additional term to independently calibrate the model against the plane jet data:

$$
I_{\omega}^{(2)}=-C_{\omega 3} 0.03 \tanh \frac{N_{\omega k}-\beta N_{k \omega}}{0.03} \omega^{2} .
$$

The dimensionless aggregate $N_{k \omega}$ is added with the negative sign to compensate the action of $N_{\omega k}$. A series of computations has been conducted with the use of $I_{\omega}^{(2)}$ with different $\beta$ values. It is found that $\eta_{0.5}=0.105$ is achieved when $\beta=1.0$; so, the final formulation of the extra source term is as follows:

$$
I_{\omega}=-C_{\omega 3} 0.03 \tanh \frac{N_{\omega}}{0.03} \omega^{2} ; \quad C_{\omega 3}=22 ; \quad N_{\omega}=N_{\omega k}-N_{k \omega}=\frac{2 \bar{\Omega}_{i j}}{\omega^{4}} \frac{\partial \omega}{\partial x_{i}} \frac{\partial k}{\partial x_{j}} .
$$

Plane jet velocity profiles obtained with (2) and without $I_{\omega}(1)$ are depicted in Fig. $5 b$. With $I_{\omega}$, the profile is slightly too sharp at the outer boundary; however, it falls within the experimental data range.

\section{COMPLETE REYNOLDS EQUATION SYSTEM COMPUTATIONS}

The SSG/LRR- $\omega$ turbulence model with the ability to enable the modifications proposed in the paper has been implemented within TsAGI in-house code de- 


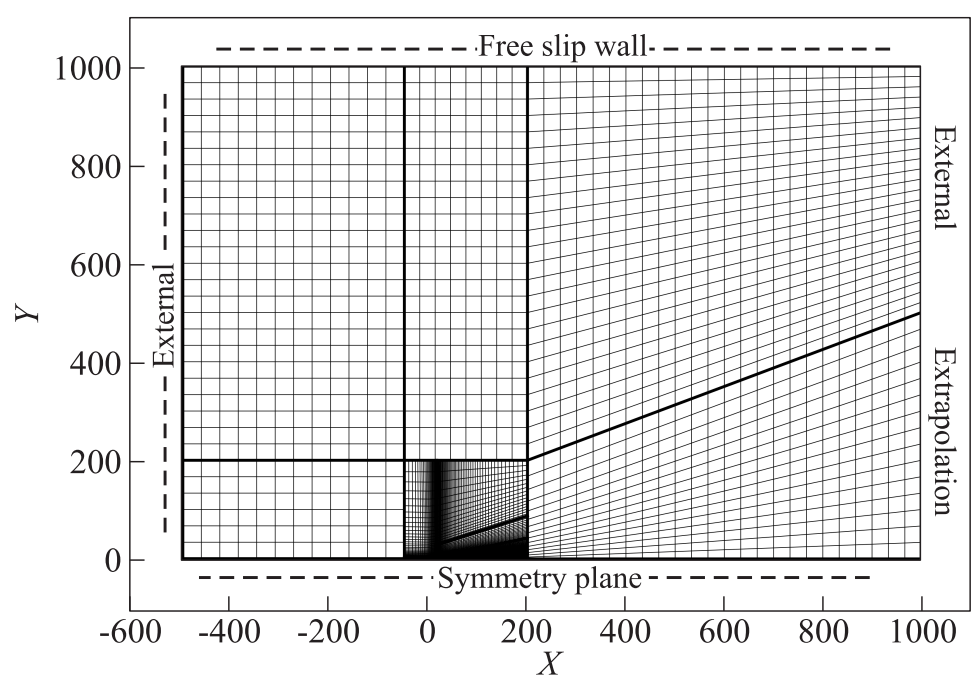

Figure 6 Computational domain, the mesh, and far-field boundary conditions in the computation of the plane jet

signed to solve the complete unsteady Favre averaged Reynolds equation system. The numerical method used in conjunction with SSG/LRR- $\omega$ model is based on explicit second-order finite-volume Godunov-Kolgan-Rodionov scheme. Steady computations are conducted with local time stepping. Multiblock structured meshes are used. For comparison, the results obtained with the $k-\omega$ shear stress transport (SST) turbulence model [13] using the same code are also presented.

A plane cold subsonic free jet has been computed. Nozzle width based Reynolds number $\operatorname{Re}=u_{0} h / \nu$ is $6.7 \cdot 10^{6}$ and Mach number is 0.30 . Computational domain, the mesh, and far-field boundary conditions are shown in Fig. 6. The region of fine mesh (of characteristic size 200h) is surrounded by buffer blocks to move the computational domain boundaries $500 h-1000 h$ far from the nozzle exit section which is positioned in the coordinate frame origin. The lower computational domain boundary is the jet symmetry plane.

Turbulent boundary layer is modeled on the internal nozzle surface and contains 25-30 cells across it. To avoid the Kelvin-Helmholtz type instability, longitudinal mesh refinement near the nozzle lip is not made. The jet mixing layer contains 60 cells across it. In the jet far field, the mesh is refined in the transverse direction so that there are 70 cells across the jet half-width. Mesh convergence study has confirmed this mesh to be enough to accurately resolve the self-similar regions of the jet.

In Fig. 7, the longitudinal velocity flow field obtained with the modified $\mathrm{SSG} / \mathrm{LRR}-\omega$ model is shown. 


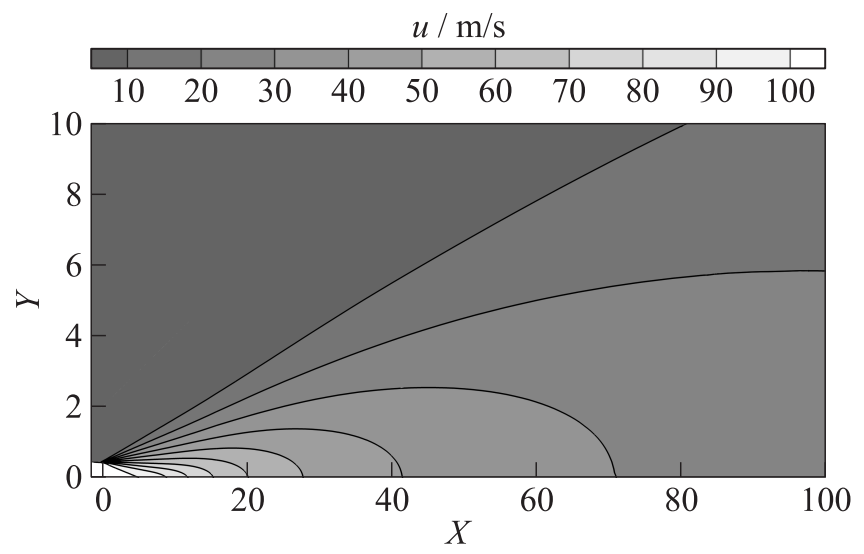

Figure 7 Longitudinal velocity flow field obtained with the modified SSG/LRR- $\omega$ model

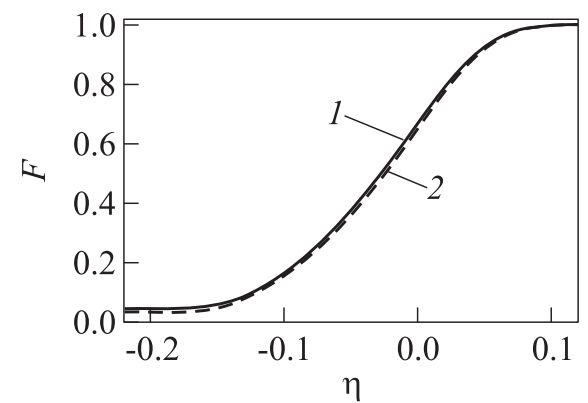

(a)

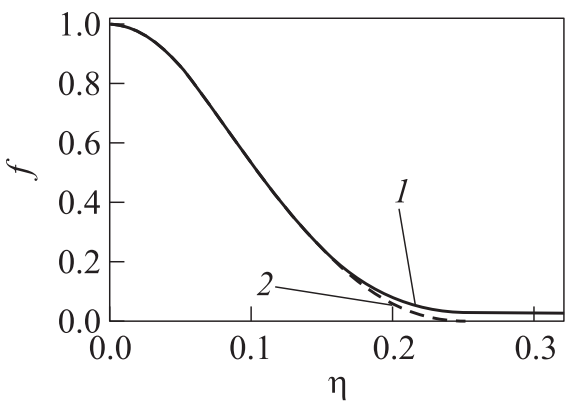

(b)

Figure 8 Velocity profiles comparison between the complete equation system (1) and self-similar approximation (2): (a) mixing layer; and (b) jet far field

The velocity profile of the jet mixing layer in $x / h=3$ cross section is compared to the self-similar profile obtained earlier in Fig. $8 a$. The far-field velocity profiles $(x / h=60$ and self-similar $)$ are depicted in Fig. $8 b$. The differences between the profiles are insignificant, which confirms the applicability of self-similar approximation to the analysis conducted in the paper. The most pronounced discrepancies are on the low-velocity boundary of the turbulent zones and are due to the absence of the vertical wall above the nozzle exit section in the complete Reynolds equation system computations.

Axial velocity $u_{c}$ distributions in the initial and part of the transitional region of the jet are shown in Fig. $9 a$. The modified model is compared to the original 


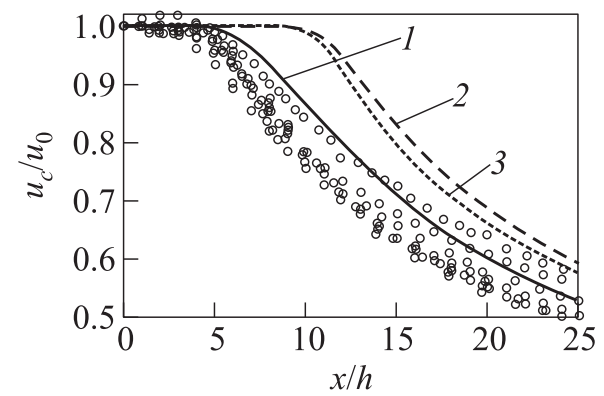

(a)

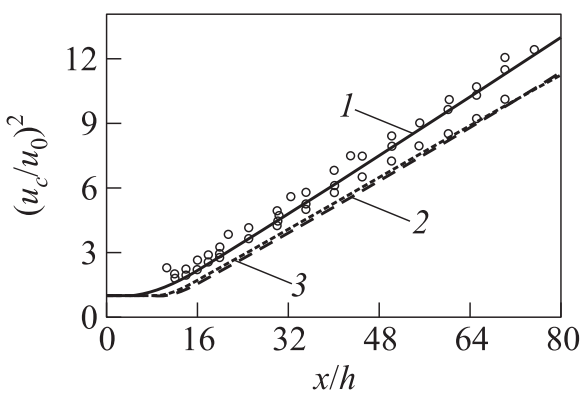

(b)

Figure 9 Axial velocity distributions: (a) $x / h \leq 20 ;(b) x / h \leq 80 ; 1$ - modified model; 2 - original model; and 3 - SST

one. It is seen that the modified model does predict the potential core length $L_{\text {ini }} \approx 6$ whereas the experimental range is $5 \leq L_{\text {ini }} \leq 6$, which means the problem of potential core length overestimation is solved. The original model overpredicts $L_{\text {ini }}$ nearly by a factor of 2 as well as the SST model.

In Fig. 9b, axial velocity distributions in the far field of the jet are presented. First, it can be observed that $\left(u_{0} / u_{c}\right)^{2}$ grows almost linearly obeying the selfsimilar relation, growth rate being in accordance with the experimental data. Second, the modified model corresponds to the experiments in $x / h>15$ region while both the original SSG/LRR- $\omega$ and the SST models shift the velocity distribution downstream.

To ensure that the proposed modifications do not negatively influence the description of boundary layers, the flat plate boundary layer computations have been conducted. Inflow Mach number is equal 0.8 and the plate is adiabatic. The length of the plate $L$ is $1.5 \mathrm{~m}$ which corresponds to $\operatorname{Re}_{L}=1.7 \cdot 10^{7}$. The outer boundaries of the computational domain are about $3 L$ far from the plate. To analyze the boundary layer velocity profile, a cross section is taken where momentum thickness Reynolds number $\operatorname{Re}_{\theta}$ is equal $16 \cdot 10^{3}$. There are approximately 100 cells across the boundary layer, the height of the first cell above the wall $y^{+}$being equal 0.1 .

Skin friction distribution along the plate is shown in Fig. 10a. Again, the original SSG/LRR- $\omega$ model (1), its modified version (2), and the SST model (3) are used. In Fig. 10b, boundary-layer velocity profile is presented in the cross section mentioned above. The experimental correlations are taken from $[14,15]$. First, all the considered models give satisfactory results. The solutions obtained with the original SSG/LRR- $\omega$ and SST models are hardly distinguishable. Second, the modified SSG/LRR- $\omega$ predicts slightly higher skin friction and alters the outer part of the boundary layer. The near-wall region is left unchanged and mostly corresponds to the experimental data. 


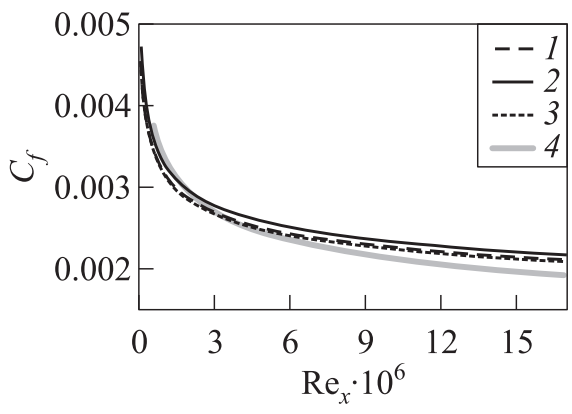

(a)

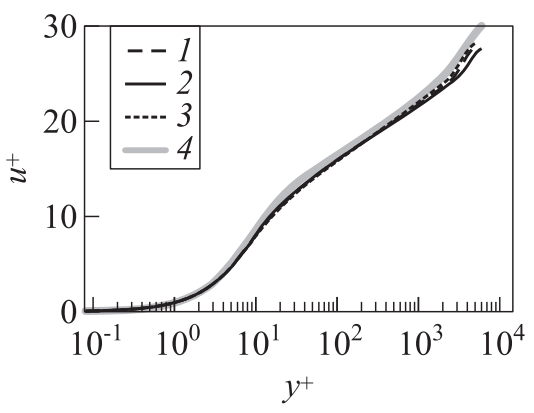

(b)

Figure 10 Computed boundary-layer characteristics: (a) skin friction along the plate; $(b)$ velocity profile at $\operatorname{Re}_{\theta}=16 \cdot 10^{3} ; 1$ - original model; 2 - modified model; 3 - SST model; and 4 - experimental correlation

\section{CONCLUDING REMARKS}

It is shown that turbulence diffusion models are not the main contributors to the discrepancies of the modern turbulence models in resolving the velocity profile of the single-stream mixing layers. Obtaining the correct solution is possible with the use of an extra source term in $\omega$ equation which accounts for the longitudinal gradients of velocity and turbulence variables and transverse velocity component connected to the gas ejection into the turbulent zone.

The proposed modifications to the SSG/LRR- $\omega$ turbulence model allow to obtain correct velocity profiles in the temporal and spatial mixing layers and in the free plane jet far field. It has been demonstrated in the computation of plane cold subsonic free jet that the modified model is superior to the original one in the whole flow field. The description of flat plate boundary layer remains almost unchanged.

The final formulation of the model is as follows:

$$
\begin{gathered}
\frac{\partial \bar{\rho} R_{i j}}{\partial t}+\frac{\partial}{\partial x_{k}}\left(\bar{\rho} R_{i j} \bar{u}_{k}-\mu \frac{\partial R_{i j}}{\partial x_{k}}-C_{R} \bar{\rho} \frac{k}{\varepsilon} R_{k l} \frac{\partial R_{i j}}{\partial x_{l}}\right)=\bar{\rho}\left(P_{i j}+\Pi_{i j}-\frac{2}{3} \varepsilon \delta_{i j}\right) ; \\
\frac{\partial \bar{\rho} \omega}{\partial t}+\frac{\partial}{\partial x_{k}}\left(\bar{\rho} \omega \bar{u}_{k}-\mu \frac{\partial \omega}{\partial x_{k}}-C_{\omega} \bar{\rho} \frac{k^{2}}{\varepsilon} \frac{\partial \omega}{\partial x_{k}}\right) \\
=\bar{\rho} \frac{\omega}{k}\left(C_{\omega 1} P-C_{\omega 2} \varepsilon\right)+\bar{\rho} I_{\omega}+\frac{\alpha_{d} C_{\omega}}{C_{\mu}} \frac{\bar{\rho}}{\omega} \max \left(\frac{\partial k}{\partial x_{k}} \frac{\partial \omega}{\partial x_{k}}, 0\right) ; \\
\varepsilon=C_{\mu} k \omega ; C_{\mu}=0.09 ; \quad I_{\omega}=-C_{\omega 3} 0.03 \tanh \frac{N_{\omega}}{0.03} \omega^{2} ; \quad N_{\omega}=\frac{2 \bar{\Omega}_{i j}}{\omega^{4}} \frac{\partial \omega}{\partial x_{i}} \frac{\partial k}{\partial x_{j}}
\end{gathered}
$$


Table 2 The modified SSG/LRR- $\omega$ model coefficients

\begin{tabular}{lccccccccccccc}
\hline Turbulence & $C_{\omega 1}$ & $C_{\omega 2}$ & $C_{\omega 3}$ & $C_{R}$ & $C_{\omega}$ & $\alpha_{d}$ & $C_{1}$ & $C_{1}^{*}$ & $C_{2}$ & $C_{3}$ & $C_{3}^{*}$ & $C_{4}$ & $C_{5}$ \\
\hline Near-wall & 0.5556 & 0.8333 & 0 & 0.0675 & 0.045 & 0 & 1.8 & 0 & 0 & 0.8 & 0 & $\frac{267}{275}$ & $\frac{159}{275}$ \\
Free & 0.48 & 0.86 & 22 & 0.20 & 0.0593 & 0.566 & 1.7 & 0.9 & 1.05 & 0.8 & 0.65 & 0.625 & 0.2 \\
\hline
\end{tabular}

The production term, redistribution term, and blending function are left unchanged and are given by formulas (3), (4), and (5), respectively.

The coefficient values are given in Table 2 .

\section{ACKNOWLEDGMENTS}

The reported study was partially supported by the Russian Foundation for Basic Research (project No. 14-01-31546).

\section{REFERENCES}

1. Engblom, W. A., N. J. Georgiadis, and A. Khavaran. 2005. Investigation of variablediffusion turbulence model correction for round jets. AIAA Paper No. 2005-3085.

2. Vlasenko, V., S. Bosniakov, S. Mikhailov, A. Morozov, and A. Troshin. 2010. Computational approach for investigation of thrust and acoustic performances of present-day nozzles. Prog. Aerosp. Sci. 46:141-197.

3. Troshin, A. I. 2012. A turbulence model with variable coefficients for calculating mixing layers and jets. Fluid Dyn. 47:320-328.

4. Cécora, R.-D., R. Radespiel, B. Eisfeld, and A. Probst. 2015. Differential Reynoldsstress modeling for aeronautics. AIAA J. 53:739-755.

5. Speziale, C. G., S. Sarkar, and T. B. Gatski. 1991. Modelling the pressure-strain correlation of turbulence: An invariant dynamical systems approach. J. Fluid Mech. 227:245-272.

6. Wilcox, D. C. 1998. Turbulence modeling for CFD. 2nd ed. La Cañada, CA: DCW Industries. 540 p.

7. Pope, S. B. 2000. Turbulent flows. Cambridge: Cambridge University Press. 802 p.

8. El Baz, A., T. J. Craft, N. Z. Ince, and B. E. Launder. 1993. On the adequacy of the thin-shear-flow equations for computing turbulent jets in stagnant surroundings. Int. J. Heat Fluid Flow 14:164-169.

9. Bell, J. H., and R. D. Mehta. 1990. Development of a two-stream mixing layer from tripped and untripped boundary layers. AIAA J. 28:2034-2042. 
10. Cazalbou, J.B., and P. Chassaing. 2002. The structure of the solution obtained with Reynolds-stress-transport models at the free-stream edges of turbulent flows. Phys. Fluids 14:597-611.

11. Cho, J. R., and M. K. Chung. 1992. A $k-\varepsilon-\gamma$ equation turbulence model. J. Fluid Mech. 237:301-322.

12. Dopazo, C. 1977. On conditioned averages for intermittent turbulent flows. J. Fluid Mech. 81:433-438.

13. Vieser, W., T. Esch, and F. Menter. 2001. Heat transfer predictions uing advanced two-equation turbulence models. CFX Technical Memorandum CFX-VAL10/0602. $73 \mathrm{p}$.

14. Schlichting, H. 1968. Boundary-layer theory. 6th ed. New York, NY: McGraw-Hill. $747 \mathrm{p}$.

15. Vlasenko, V. V., and A. A. Shiryaeva. 2013. Numerical simulation of non-stationary propagation of combustion along a duct with supersonic flow of a viscid gas. Proc. Inst. Mech. Eng. G J. Aerosp. Eng. 227(3):480-492. 